\title{
Physicochemical and Biological Evaluation of siRNA Polyplexes Based on PEGylated Poly(amido amine)s
}

\author{
Pieter Vader • Leonardus J. van der Aa • Johan F. J. Engbersen • Gert Storm • Raymond M. Schiffelers
}

Received: 30 May 2011 / Accepted: 21 July 2011 / Published online: 11 August 2011

(C) The Author(s) 2011. This article is published with open access at Springerlink.com

\begin{abstract}
Purpose Use of RNA interference as novel therapeutic strategy is hampered by inefficient delivery of its mediator, siRNA, to target cells. Cationic polymers have been thoroughly investigated for this purpose but often display unfavorable characteristics for systemic administration, such as interactions with serum and/or toxicity.

Methods We report the synthesis of a new PEGylated polymer based on biodegradable poly(amido amine)s with disulfide linkages in the backbone. Various amounts of PEGylated polymers were mixed with their unPEGylated counterparts prior to polyplex formation to alter PEG content in the final complex.

Results PEGylation effectively decreased polyplex surface charge, salt- or serum-induced aggregation and interaction with erythrocytes. Increasing amount of PEG in formulation also reduced its stability against heparin displacement, cellular uptake and subsequent silencing efficiency. Yet, for polyplexes with high PEG content, significant gene silencing efficacy was found, which was combined with almost no toxicity.
\end{abstract}

Conclusions PEGylated poly(amido amine)s are promising carriers for systemic siRNA delivery in vivo.

P. Vader $\cdot$ G. Storm $\cdot$ R. M. Schiffelers

Department of Pharmaceutics

Utrecht Institute for Pharmaceutical Sciences, Utrecht University

P.O. Box 80082, 3508 TB Utrecht, The Netherlands

L. J. van $\operatorname{der} A a \cdot J$. F. J. Engbersen

Department of Biomedical Chemistry

MIRA Institute for Biomedical Technology \& Technical Medicine

Faculty of Science \& Technology, University of Twente

P.O. Box 217, 7500 AE Enschede, The Netherlands

P. Vader $(\bowtie)$

Universiteitsweg 99

3584 CG Utrecht, The Netherlands

e-mail: P.Vader@uu.nl
KEY WORDS biodegradable · delivery . PEGylation · poly (amido amine)s · siRNA

\section{INTRODUCTION}

RNA interference (RNAi) using small interfering RNAs (siRNAs) has emerged as a powerful tool for knockdown of genes and holds great potential as a novel therapeutic strategy for a broad range of diseases $(1,2)$. The primary limitation to clinical success, however, is the lack of efficient delivery systems that can deliver siRNA to the target cell population. siRNAs do not readily pass cellular membranes due to their negative charge and are rapidly degraded by serum nucleases.

Cationic polymers have widely been investigated for siRNA delivery due to their great flexibility, ease of manufacturing and modification possibilities (3). Typical examples include poly(ethyleneimine) (pEI) (4), poly(Llysine) (pLL) (5), chitosan (6) and poly(2-(dimethyl-amino) ethylmethacrylate) (pDMAEMA) (7). Although significant progress has been made in improving these polymers for siRNA delivery, either low efficiency and/or high cytotoxicity remain to hinder their usefulness. Recently, we have described a new class of biodegradable polymers based on poly(amido amine)s with disulfide linkages in the backbone (SS-PAA) that were specifically tailored for delivery of siRNA $(8,9)$. These copolymers were composed of $\mathcal{N}, \mathcal{N}^{\prime}$ cystaminebisacrylamide (CBA), 4-amino-1-butanol (ABOL) and ethylene diamine (EDA) (p(CBA-ABOL/EDA)) and were able to complex siRNA into positively charged polyplexes that were efficiently taken up by cells and induced target gene silencing. Moreover, this was combined with low cellular toxicity, which encouraged us to perform further functional studies. 
For cancer therapy, in order to reach distant tumors or metastases, systemic administration of siRNA polyplexes is inevitable. Upon intravenous injection however, positively charged polyplexes might potentially interact aspecifically with serum proteins or erythrocytes and other blood cells, leading to formation of aggregates, which causes rapid clearance by the reticulo-endothelial systems (RES) and sometimes significant toxicity (10). The biocompatibility of polyplexes can be enhanced by conjugation of poly(ethylene glycol) (PEG) to the cationic polymer (PEGylation). Complexation of oligonucleotides with PEG-containing copolymers leads to the formation of particles with a core-shell structure, in which the cationic polymer packs the oligonucleotide within the particle core and the hydrophilic, noncharged PEG chains form a shell layer around it (11). In general, PEGylation of polyplexes results in a lower surface charge, reduced interaction with blood components, prolonged blood circulation and lower cytotoxicity $(12,13)$.On the other hand, steric shielding of the polyplex particles also leads to reduced cellular association and uptake, diminished endosomal escape properties, and inefficient siRNA release $(14,15)$. These contrasting effects associated with the use of PEG in oligonucleotide delivery are also referred to as the 'PEG dilemma' (16), and raise the need for strategies to fine-tune the PEG content in polyplexes to optimize their physicochemical and biological properties. Recently, Brumbach et al. demonstrated the feasibility of altering and optimizing PEG content in polyplexes by formulating mixtures of a polycation and its corresponding PEGylated counterpart before complex formation, avoiding the need to synthesize multiple copolymers with varying degrees of PEGylation to identify optimal carrier candidates (17).

In this study we synthesized a new PEGylated polymer, $\mathrm{p}$ (CBA-ABOL/EDA/PEG), based on the successful $\mathrm{p}(\mathrm{CBA}-\mathrm{ABOL} / \mathrm{EDA})$ polymer and used mixtures of the PEGylated and unPEGylated polymer in order to vary the PEG content in the final polyplex. Polyplexes with different PEG content were compared to corresponding unPEGylated complexes regarding physicochemical characteristics, stability, cellular uptake, gene silencing activity and in vitro biocompatibility.

\section{MATERIALS AND METHODS}

\section{Materials}

All chemicals, 4-amino-1-butanol (ABOL, Merck), ethylene diamine (EDA, Sigma-Aldrich), $\mathcal{N}, \mathcal{N}^{\prime}$-cystaminebisacrylamide (CBA, Polysciences) and $\alpha$-amino- $\omega$-hydroxy poly(ethylene glycol) (PEG-NH $2,3000 \mathrm{~g} / \mathrm{mol}$, Iris Biotech $\mathrm{GmbH}$ ) were purchased in the highest purity and used without further purification.

\section{siRNAs}

siRNAs were chemically synthesized and supplied by Eurogentec (Maastricht, The Netherlands). Sequence of siRNA against luciferase (siLuc) was 5'-GAU-UAU-GUCCGG-UUA-UGU-AUU-3' (sense) and 5'-UAC-AUAACC-GGA-CAU-AAU-CUU-3' (antisense). For cellular uptake studies, Alexa-488-modified siRNA was used (dye was attached to the 5'-end of the sense strand). Universal negative control siRNA (siNS) was purchased from Eurogentec.

\section{Polymer Synthesis and Characterization}

Synthesis of $\mathrm{p}(\mathrm{CBA}-\mathrm{ABOL} / \mathrm{EDA})$ was performed as previously described (9). p(CBA-ABOL/EDA/PEG) was synthesized by Michael addition polymerization of ABOL, EDA and PEG-NH${ }_{2}$ with $\mathcal{N}, \mathcal{N}^{\prime}$-cystaminebisacrylamide (CBA). Therefore, $424 \mathrm{mg}$ (1.63 mmol) CBA, $98 \mathrm{mg}$ (1.10 mmol) ABOL and $500 \mathrm{mg}(0.17 \mathrm{mmol}) \mathrm{PEG}-\mathrm{NH}_{2}$ were dissolved in methanol/water $(4: 1 \mathrm{v} / \mathrm{v})$ and were allowed to react at $45^{\circ} \mathrm{C}$ in the dark in a nitrogen atmosphere. The reaction mixture became homogeneous within $1 \mathrm{~h}$. After 6 days of prepolymerization, $22 \mathrm{mg}(0.37 \mathrm{mmol})$ EDA was added and the reaction was proceeded for another 2 days. Then the polymerization was terminated by addition of a $10 \%$ molar excess EDA, to consume remaining toxic acrylamide endgroups. After termination, the reaction mixture was diluted with hydrochloric acid ( $1 \mathrm{M}$ ) and water, purified by ultrafiltration (MWCO 5000, pH 5) and recovered as its $\mathrm{HCl}$ salt by lyophilization. ${ }^{1} \mathrm{H} \mathrm{NMR}\left(\mathrm{D}_{2} \mathrm{O}\right) \delta(\mathrm{ppm})=1.58$ (m, 2H, $\left.\boldsymbol{C H}_{2} \mathrm{CH}_{2} \mathrm{NR}\right) ; 1.79\left(\mathrm{~m}, 2 \mathrm{H}, \boldsymbol{C H}_{2} \mathrm{CH}_{2} \mathrm{OH}\right) ; 2.69(\mathrm{t}$, $\left.4 \mathrm{H}, \mathrm{NH}_{\boldsymbol{C}} \boldsymbol{H}_{2} \boldsymbol{C} \boldsymbol{H}_{2} \mathrm{NH}\right) ; 2.77$ (t, 2H, $\left.\boldsymbol{C H}_{2} \mathrm{CONH}\right) ; 2.83(\mathrm{t}$, $\left.4 \mathrm{H}, \boldsymbol{C} \boldsymbol{H}_{2} \mathrm{SS} \boldsymbol{C} \boldsymbol{H}_{2}\right) ; 3.22\left(\mathrm{t}, 2 \mathrm{H}, \mathrm{CH}_{2} \boldsymbol{C H}_{2} \mathrm{NR}\right) ; 3.32(\mathrm{t}, 2 \mathrm{H}$, $\left.\mathrm{COCH}_{2} \boldsymbol{C H}_{2} \mathrm{NH}\right) ; 3.44\left(\mathrm{t}, 4 \mathrm{H}, \boldsymbol{C} \boldsymbol{H}_{2} \mathrm{NR} \boldsymbol{C} \boldsymbol{H}_{2}\right) ; 3.51$ (t, 4H, $\left.\mathrm{NH}_{\boldsymbol{C}} \boldsymbol{H}_{2} \mathrm{CH}_{2} \mathrm{SSCH}_{2} \boldsymbol{C H}_{2} \mathrm{NH}\right) ; 3.60$ (t, 2H, $\left.\boldsymbol{C} \boldsymbol{H}_{2} \mathrm{OH}\right) ; 3.65-$ 3.90 (m, 272H, OC $\boldsymbol{H}_{2} \boldsymbol{C H}_{\mathbf{2}} \mathrm{O}$ ). Polymers were characterized by ${ }^{1} \mathrm{H}$ NMR $\left(\mathrm{D}_{2} \mathrm{O}\right)$, recorded on a Varian Innova spectrometer $(300 \mathrm{MHz})$. Molecular weights were determined by GPG relative to PEG standards, using a GPCmax with an acetate buffer pH 4.5 containing 30\% (v/v) methanol as eluent.

\section{Polyplex Formation}

To prepare polyplexes at different polymer/siRNA (w/w) ratios, appropriate amounts of polymer and siRNA were each diluted in glucose-containing Hepes buffer (HBG: 20 mM Hepes, pH 7.4, 5 wt. \% glucose). Next, polymer solution was added to siRNA solution (4:1, v/v), followed 
by $5 \mathrm{~s}$ vortexing and $30 \mathrm{~min}$ incubation at room temperature. To obtain polyplexes with different PEG content, expressed as the weight percentage of PEG to total polymer, polymer solutions of $\mathrm{p}(\mathrm{CBA}-\mathrm{ABOL} / \mathrm{EDA})$ and $\mathrm{p}(\mathrm{CBA}-\mathrm{ABOL} / \mathrm{EDA} / \mathrm{PEG})$ were mixed prior to polyplex formation.

\section{Polyplex Characterization}

For gel retardation assays, polyplexes were prepared in $50 \mu \mathrm{l}$ HBG at a final siRNA concentration of $2.5 \mu \mathrm{M}$ and incubated for $1 \mathrm{~h}$ at $37^{\circ} \mathrm{C}$ in the presence or absence of $5 \mathrm{mM}$ glutathione. After addition of loading dye (Fermentas, St. Leon-Rot, Germany), samples were run on a $4 \%$ agarose gel containing $0.5 \mu \mathrm{g} / \mathrm{ml}$ ethidium bromide at $90 \mathrm{~V}$ for $10 \mathrm{~min}$. Hydrodynamic diameters and $\zeta$-potentials were measured as previously described (8).

\section{Complex Aggregation in Salt}

Polyplexes were prepared in $400 \mu \mathrm{l} \mathrm{HBG}$ at a final siRNA concentration of $500 \mathrm{nM}$. Then, $2 \mathrm{ml}$ PBS was added and hydrodynamic diameters were measured at indicated time points as described above.

\section{Complex Aggregation in Serum}

Polyplexes containing were prepared in $50 \mu \mathrm{l} \mathrm{HBG}$ at a final siRNA concentration of $15 \mu \mathrm{M}$. $180 \mu \mathrm{l}$ fetal bovine serum (FBS) was added to $20 \mu \mathrm{l}$ polyplex solution and samples were incubated for $10 \mathrm{~min}$ at $37^{\circ} \mathrm{C}$. Aggregation in terms of turbidity increase of each sample was quantified by absorbance detection at $595 \mathrm{~nm}$.

\section{Stability of Polyplexes Against Heparin}

Polyplexes were prepared in $50 \mu \mathrm{l} \mathrm{HBG}$ at a final siRNA concentration of $2.5 \mu \mathrm{M}$ and incubated for $30 \mathrm{~min}$ with heparin solutions of different concentrations, expressed as heparin/siRNA (w/w) ratio. After addition of loading dye, samples were run on a $4 \%$ agarose gel containing $0.5 \mu \mathrm{g} / \mathrm{ml}$ ethidium bromide at $90 \mathrm{~V}$ for $10 \mathrm{~min}$.

\section{Erythrocyte Aggregation and Hemolysis}

Erythrocytes were obtained from $200 \mu$ l whole murine blood by multiple centrifugation rounds $\left(1000 \mathrm{~g}, 10 \mathrm{~min}, 4^{\circ} \mathrm{C}\right.$ ) followed by washing the pellet in PBS until the supernatant was clear. The final pellet was resuspended in $4 \mathrm{ml}$ PBS. Subsequently, $160 \mu \mathrm{l}$ erythrocyte suspension was added to $40 \mu \mathrm{l}$ polyplex solution having a siRNA concentration of $4 \mu \mathrm{M}$ and samples were incubated for $1 \mathrm{~h}$ at $37^{\circ} \mathrm{C}$. Triton
X-100 (1\%) in PBS (100\% lysis) and HBG (0\% lysis) were used as controls. After a final centrifugation step, $150 \mu \mathrm{l}$ of the supernatant was analyzed for hemoglobin release by absorbance detection at $550 \mathrm{~nm}$. The pellet was resuspended in $50 \mu \mathrm{l}$ PBS for microscopic evaluation using a Nikon TE2000-U microscope.

\section{Cell Culture}

H1299 (human lung cancer) cells stably expressing firefly luciferase were cultivated at $37^{\circ} \mathrm{C}$ and $5 \% \mathrm{CO}_{2}$ in RPMI 1640 (PAA laboratories GmbH, Pasching, Austria) supplemented with $10 \%$ (v/v) FBS, $100 \mathrm{IU} / \mathrm{ml}$ penicillin, $100 \mu \mathrm{g} / \mathrm{ml}$ streptomycin and $0.25 \mu \mathrm{g} / \mathrm{ml}$ amphotericin B.

\section{Cellular Uptake}

Quantification of siRNA uptake was performed as previously described, with minor modifications (18). H1299 cells were seeded in 6 -well plates at a density of $1.6 \times 10^{5}$ cells per well, $24 \mathrm{~h}$ before transfection. Cells were treated with $400 \mu \mathrm{l}$ of different polyplexes containing Alexa-488 labeled siRNA at a final siRNA concentration of $125 \mathrm{nM}$ in FBScontaining medium. After $4 \mathrm{~h}$, cells were washed twice with PBS to remove non-internalized polyplexes. $300 \mu \mathrm{l}$ lysis buffer $(2 \%$ SDS, $1 \%$ Triton X-100 in PBS) was added to lyse the cells and dissociate polyplexes. Cells were lysed for $1 \mathrm{~h}$ on ice, after which the lysates were centrifuged $(14000 \mathrm{~g}$, $15 \mathrm{~min}, 4^{\circ} \mathrm{C}$ ) to remove cell debris. $200 \mu \mathrm{l}$ supernatant was transferred to a black 96-well plate to measure fluorescence using a Fluostar OPTIMA microplate-based multidetection reader (Isogen Life Science, Maarssen, The Netherlands). The mean fluorescence intensity was normalized to the amount of protein present in the sample, determined using the MicroBCA ${ }^{\mathrm{TM}}$ protein assay (Pierce, Rockford, USA).

For microscopy, cells were seeded in 12-well plates on coverslips, $24 \mathrm{~h}$ before transfection. Cells were treated with $20 \mu \mathrm{l}$ of different polyplexes containing Alexa-488 labeled siRNA at a final siRNA concentration of $125 \mathrm{nM}$. After $4 \mathrm{~h}$, cells were washed with PBS and fixed with $4 \%$ paraformaldehyde in PBS at room temperature for $30 \mathrm{~min}$. After fixation, slides were washed, counterstained with DAPI and mounted using Fluorsave (Calbiochem, San Diego, CA, USA). Cells were imaged using a Nikon TE2000-U fluorescent microscope (Nikon Benelux, Brussels, Belgium).

\section{In Vitro Gene Silencing}

H1299 cells were seeded in 96-well plates at a density of 8000 cells per well, $24 \mathrm{~h}$ before transfection. Cells were 
treated with $20 \mu \mathrm{l}$ of different polyplexes at a final siRNA concentration of $125 \mathrm{nM}$ in FBS-containing medium. After $4 \mathrm{~h}$, medium was replaced, and cells were incubated for another $48 \mathrm{~h}$. Then, cells were washed and lysed in $100 \mu \mathrm{l}$ reporter gene lysis buffer (Promega, Leiden, The Netherlands). After a freeze/ thaw cycle, $50 \mu \mathrm{l}$ lysate was mixed with $50 \mu \mathrm{l}$ luciferase assay reagent (Promega) and luciferase activity was determined by measuring the luminescence for $10 \mathrm{~s}$ at room temperature using a Fluostar OPTIMA microplate-based multi-detection reader equipped with a microinjector (Isogen Life Science). Luciferase activity of untreated cells was defined as $100 \%$ expression.

\section{Statistical Analysis}

Results were analyzed using Student's $t$-tests to assess statistical significances. For multiple comparisons, ANOVA with a Bonferroni post-test was used.

\section{RESULTS AND DISCUSSION}

\section{Polymer Synthesis}

Two reducible poly(amido amine) copolymers were synthesized by Michael-type addition polymerization from $\mathcal{N}, \mathcal{N}^{\prime}$ cystaminebisacrylamide (CBA), 4-amino-1-butanol (ABOL) and ethylene diamine (EDA) with or without $\alpha$-amino- $\omega$ hydroxy poly(ethylene glycol) $\left(\mathrm{PEG}-\mathrm{NH}_{2}\right)$, to obtain a PEGylated and a non-PEGylated polymer (Fig. 1). All polymers were obtained as a white to slightly yellow brittle material. The molar ratio between ABOL and EDA was found to be optimal at 75/25 for the unPEGylated polymer (9) and this ratio was used again here. The characteristics of the final polymers are listed in Table 1 . The obtained ratio between ABOL, EDA and PEG, as well as the PEG content were determined from the NMR spectra. Molecu- lar weights were determined by GPC. The obtained ratio between $\mathrm{ABOL} / \mathrm{EDA}$ in the polymers deviated only slightly from the feed ratio and the obtained PEG fraction in the PEGylated polymer corresponded well with the aimed fraction. Based on the previous results in the $\mathrm{p}(\mathrm{CBA}$ $\mathrm{ABOL} / \mathrm{EDA}$ ) synthesis (9), all polymers are expected to be linear.

\section{Polyplex Characteristics}

In order to prepare polyplexes with various amounts of PEG in the formulations, mixtures containing different ratios of $\mathrm{p}(\mathrm{CBA}-\mathrm{ABOL} / \mathrm{EDA})$ and $\mathrm{p}(\mathrm{CBA}-\mathrm{ABOL} / \mathrm{EDA} /$ PEG) were taken and mixed with siRNA. As it has previously been shown that PEG conjugation to polymeric vectors can interfere with polyplex formation and siRNA complexation, we first investigated the physicochemical properties of polyplexes with different PEG contents.

To determine the ability of the polymer mixtures to complexate siRNA, an agarose gel retardation assay was performed. Polyplexes were formed at polymer/siRNA $(\mathrm{w} / \mathrm{w})$ ratios of 0 (free siRNA), 3, 6, 12, 24 and 48. In the determination of the $\mathrm{w} / \mathrm{w}$ ratios, the weight of PEG was not taken into account, thus the given ratios reflect the relative amount of cationic polymer in each formulation. For unPEGylated polyplexes, total siRNA retardation was observed at w/w ratios 12 and higher, indicating that the nucleic acid remained associated with the cationic polymers that do not migrate into the gel (Fig. 2a, left). Complete retardation at this $\mathrm{w} / \mathrm{w}$ ratio was also observed for polyplexes with 15, 30 and $45 \mathrm{wt} . \%$ PEG (Fig. 2b-d, left). For polyplexes that were prepared with $45 \mathrm{wt} . \%$ PEG at the lower w/w ratios 3 and 6 , siRNA was still visible in the wells, indicating that it was bound to the polymer but not completely shielded as ethidium bromide was still able to intercalate (Fig. 2d, left).

The disulfide bridges in the poly(CBA-ABOL/EDA) polymers are expected to be cleaved in the cytosol because
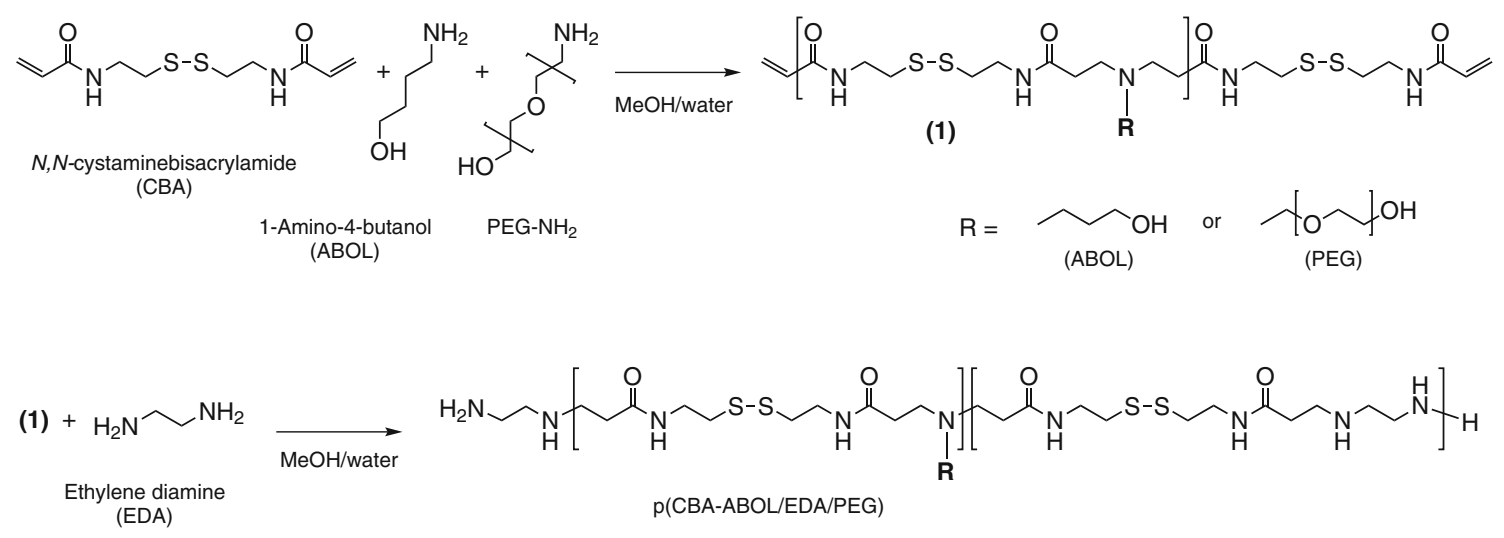

Fig. I Schematic representation of the synthesis of the $p(C B A-A B O L / E D A)$ and $p(C B A-A B O L / E D A / P E G)$ copolymers. 
Table I Synthesis Characteristics

\begin{tabular}{|c|c|c|c|c|c|c|}
\hline Polymer & $\begin{array}{l}\text { Feed composition }^{\text {a }} \\
\text { ABOL/EDA/PEG }\end{array}$ & $\begin{array}{l}\text { Obtained composition } \\
\text { ABOL/EDA/PEG }\end{array}$ & Wt \% PEG ${ }^{\mathrm{c}}$ & $M_{w}(\mathrm{~kg} / \mathrm{mol})$ & PDI & $M_{w}$ PAA $(\mathrm{kg} / \mathrm{mol})^{d}$ \\
\hline$p(C B A-A B O L / E D A)$ & $75 / 25 / 0$ & $80 / 20 / 0$ & $0 \%$ & 11.6 & 2.4 & 11.6 \\
\hline$p(C B A-A B O L / E D A / P E G)$ & $67 / 23 / 10$ & $59 / 31 / 10$ & $52 \%$ & 47.8 & 1.4 & 23.9 \\
\hline
\end{tabular}

${ }^{\text {a }}$ Stoichiometrical feed ratio of monomers

${ }^{\mathrm{b}}$ Determined by ${ }^{1} \mathrm{H}$ NMR

c $P E G$ fraction of the polymer expressed as a weight percentage

${ }^{d}$ Molecular weight of the PAA fraction in the polymer, calculated from the wt.\% PEG

of its high redox potential as compared to the extracellular environment. To investigate whether the accessibility of these disulfide bonds for reducing agents like glutathione is restricted by $\mathrm{PEG}$ ylation of the polyplexes, polyplexes were incubated for $1 \mathrm{~h}$ at $37^{\circ} \mathrm{C}$ in the presence of $5 \mathrm{mM}$ glutathione and subjected to electrophoresis. For all formulations, this incubation resulted in total release of siRNA from the polyplexes, indicating that the PEG chains do not interfere with polymer reduction (Fig. 2a-d, right). Based on these results, further studies were performed with polyplexes prepared at polymer/siRNA w/w ratio 24 .

The influence of PEGylation on polyplex size and surface charge was investigated using dynamic light scattering and laser Doppler electrophoresis, respectively. UnPEGylated polyplexes prepared at w/w ratio 24 were around $160 \mathrm{~nm}$ in diameter and positively charged $( \pm 20 \mathrm{mV})$ (Fig. 3a, b). Polyplexes with 15 wt. $\%$ or 30 wt. $\%$ PEG were around the same size, while polyplexes formed with $45 \mathrm{wt} . \%$ PEG were slightly smaller (Fig. 3a). Smaller sizes for PEGylated polyplexes as compared to their unPEGylated counterparts has been shown before and was contributed to effective shielding and subsequent abolished aggregation

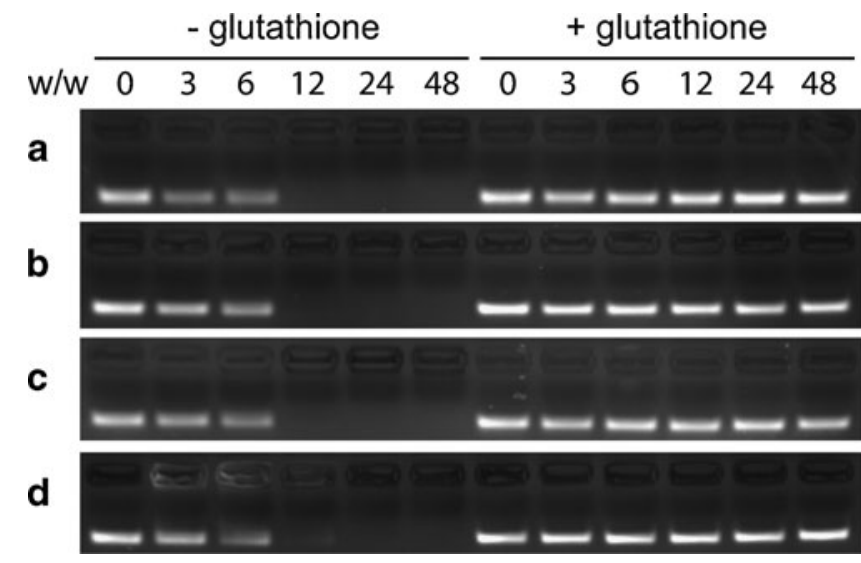

Fig. 2 Agarose gel retardation of polyplexes prepared at w/w ratios 0 (siRNA only) to 48 , after $\mathrm{h}$ incubation at $37^{\circ} \mathrm{C}$ in the absence or presence of $5 \mathrm{mM}$ glutathione. Polyplexes were prepared using polymers without PEG (a) or with a PEG content of 15 wt.\% (b), 30 wt.\% (c) and 45 wt. \% (d)
$(19,20)$. Increased size for PEGylated polyplexes has also been reported, but always linked to perturbed nucleic acid condensation (17,21). However, together with the data obtained from the agarose gel retardation assay, these results show that PEG conjugation of these polymers does not negatively affect polyplex formation. Furthermore, PEGylation substantially decreased polyplex surface charge down to $\pm 5 \mathrm{mV}$ for polyplexes with 45 wt. $\%$ PEG, which is in accordance with other reports on effects of polyplex PEGylation (17,22,23) (Fig. 3b).
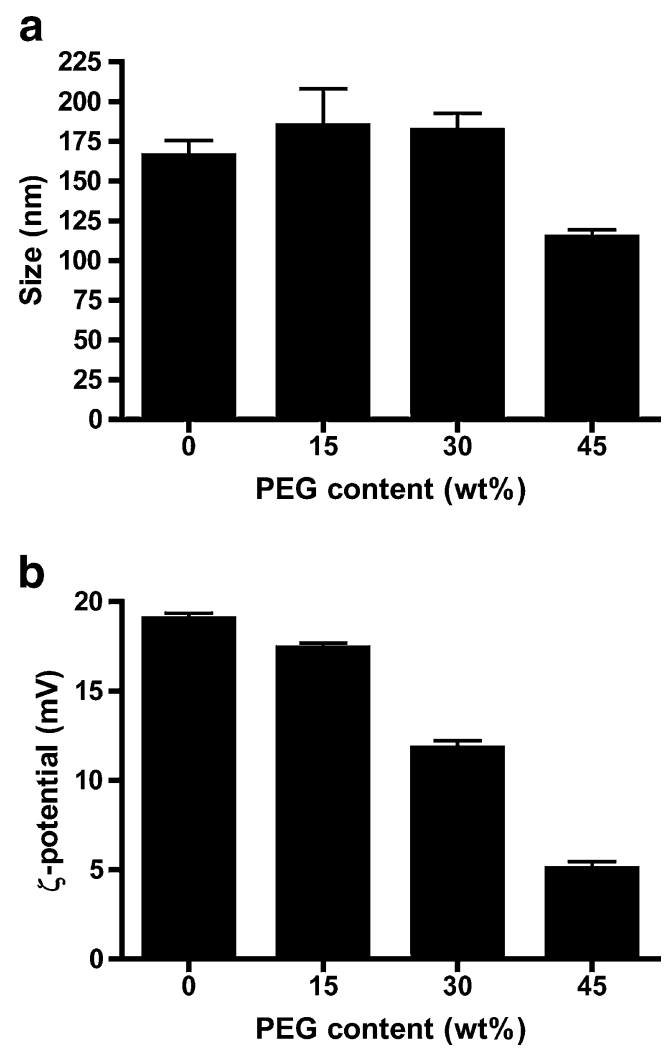

Fig. 3 Size (a) and $\zeta$-potential (b) of polyplexes prepared at polymer/ siRNA (w/W) ratio 24 using polymers with increasing PEG content, as determined by dynamic light scattering and laser Doppler electrophoresis. Results are shown as mean \pm SD for three individual measurements. 


\section{Polyplex Stability}

Systemic administration exposes polyplexes to physiological ionic strengths and anionically charged proteins, which can induce aggregation and destabilization by non-specific interactions (13). To investigate whether PEGylation prevents polyplex aggregation at physiological salt concentrations, pre-formed polyplexes were incubated in PBS and size measurements were performed over time using dynamic light scattering. As expected, polyplexes without PEG showed a gradual increase in size to approximately $800 \mathrm{~nm}$ after $4 \mathrm{~h}$ due to aggregation (Fig. 4). Increasing the PEG content resulted in enhanced stabilization of the polyplexes. Polyplexes containing 15 wt. \% PEG displayed an aggregation profile similar to that of polyplexes without PEG, whereas a content of 30 wt.\% PEG substantially inhibited aggregation. 45 wt. $\%$ PEG totally prevented aggregation up to $4 \mathrm{~h}$. Similar PEG-density dependent stabilization profiles were found for post-PEGylated cyclodextrin-based polyplexes formed with siRNA (24) or pDNA (25). Next, we assessed effects of PEGylation on serum-induced aggregation by incubating pre-formed polyplexes in 90\% FCS. Aggregation in terms of turbidity increase was significantly prevented by coupling of PEG to the polymeric structures and appeared to be more pronounced with increasing PEG content (Fig. 5). Combined, these results indicate that PEGylation prevents polyplex aggregation in biological media. Serum proteins as well as extracellular matrix components can however, besides inducing aggregation, also lead to vector disassembly (26). To evaluate possible effects of PEG on polyplex stability against competing anions, we tested the susceptibility of our polyplexes to heparin displacement. Heparin is one of the glycosaminoglycans (GAG), which are negatively charged polysaccharides that are the major components of the extracellular matrix in many tissues and are also found on

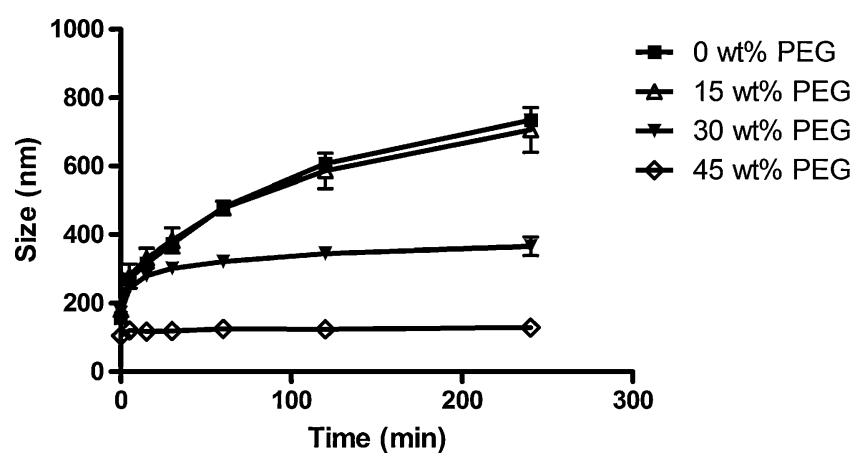

Fig. 4 Polyplex stability in a physiological salt solution. Polyplexes were prepared at polymer/siRNA (w/w) ratio 24 using polymers with increasing PEG content. Particle sizes were determined by dynamic light scattering. Results are shown as mean \pm SD for two individual measurements.

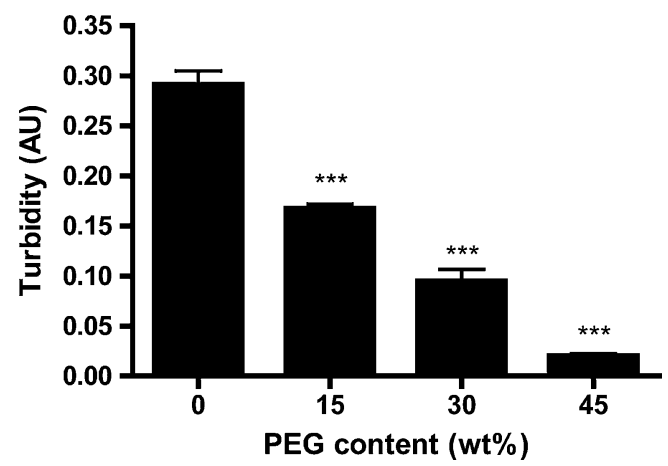

Fig. 5 Serum-induced aggregation of polyplexes. Polyplexes were prepared at polymer/siRNA ( $W / W$ ) ratio 24 using polymers with increasing PEG content. Aggregation in $90 \%$ FCS in terms of turbidity increase was quantified by absorbance detection at $595 \mathrm{~nm}$. Results are shown as mean \pm SD for $n=3$. Statistically significant differences versus polyplexes without PEG are denoted by $* * * * * 0.00 I)$

the cell surface (27). Polyplexes with different PEG contents were prepared and subsequently incubated with increasing heparin concentrations. Polyplex dissociation in terms of siRNA release was shown by electrophoresis of the samples on an ethidium bromide-containing agarose gel (Fig. 6). For unPEGylated polyplexes, dissociation occurred at heparin/siRNA $(\mathrm{w} / \mathrm{w})$ ratio 4 and higher. The presence of 15 wt.\% PEG did not alter the stability against heparin, but for polyplexes with higher PEG content a slightly decreased resistance against heparin competition was observed. Such a negative effect of PEG on polyplex stability was reported earlier for pEI-PEG/siRNA polyplexes and contributed to the weakened interaction with siRNA in the case of the PEGylated polymer as a result of decreased charge density (23).

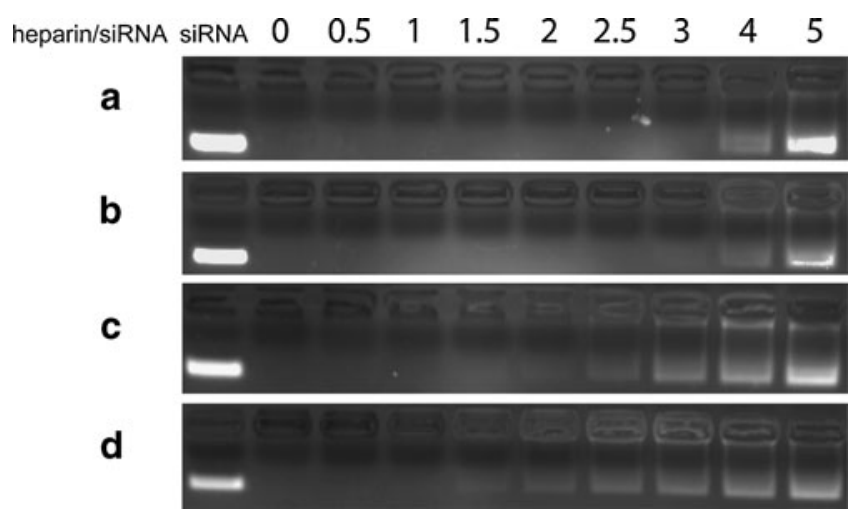

Fig. 6 Heparin displacement assay. Polyplexes were prepared at w/w ratio 24 and incubated for $30 \mathrm{~min}$ at $37^{\circ} \mathrm{C}$ in the presence of increasing amounts of heparin, expressed as heparin/siRNA (w/W) ratio. Polyplexes were prepared using polymers without PEG (a) or with a PEG content of 15 wt.\% (b), 30 wt. \% (c) and 45 wt.\% (d). 


\section{Erythrocyte Aggregation and Hemolysis}

Besides interactions with salt, proteins and/or the extracellular matrix, interactions with blood cells such as erythrocytes occur after systemic injection. Erythrocytes bear a negative surface charge and are known to interact with positively charged polyplexes, leading to aggregate formation and systemic toxicity (28). To evaluate the influence of PEG on polyplex-induced erythrocyte aggregation and hemolysis, polyplexes with different PEG content were incubated for $1 \mathrm{~h}$ with freshly purified red blood cells. Erythrocytes were pelleted and visualized under a microscope, while the supernatant was used for measuring the degree of hemolysis as determined by absorbance detection at $550 \mathrm{~nm}$. Control polyplexes were prepared using poly(2(dimethylamino)ethyl methacrylate) (pDMAEMA), which were previously shown to induce severe erythrocyte aggregation (29). In our experiments, erythrocytes incubated with pDMAEMA polyplexes also displayed obvious aggregation as compared to cells incubated with buffer only. Polyplexes prepared from $\mathrm{p}(\mathrm{CBA}-\mathrm{ABOL} / \mathrm{EDA})$ polymers induced only mild aggregation, which was even further decreased using PEGylated polymers. For polyplexes containing 30 or 45 wt. $\%$ PEG, no erythrocyte aggregation was observed (Fig. 7a). A similar trend was observed for the degree of hemolysis. For polyplexes formed with pDMAEMA, approximately $16 \%$ hemolysis was observed, similar to what others have found for pEI polyplexes (30). A much lower hemolytic activity was measured for pCBA-ABOL/EDA polyplexes (approx. $4 \%$ ). For PEGylated polyplexes, even less hemolysis was observed, down to less than $1 \%$ hemolysis for polyplexes with 45 wt. \% PEG (Fig. 7b). Together, these data indicate that PEGylated polymers have reduced interactions with blood components, which is in accordance with findings from others $(13,31)$.

\section{Cellular Uptake and Gene Silencing}

To study the effects of PEGylation on intracellular uptake of polyplexes, H1299 cells were transfected with polyplexes with increasing PEG content, formulated using Alexa488labeled siRNA. For quantification of siRNA internalization, fluorescence was determined in cell lysates $4 \mathrm{~h}$ after transfection. 2\% SDS was added to the lysis buffer to ensure complete polyplex dissociation, preventing possible quenching effects (18). As shown in Fig. 8a, for all polymers, uptake was less than for control lipoplexes based on Lipofectamine 2000. Cellular uptake decreased with increasing PEG content in the polyplex. While the extent of uptake for polyplexes with 15 wt.\% PEG was similar to that of unPEGylated polyplex, uptake was significantly reduced for polyplexes containing $30 \mathrm{wt} . \%$ PEG and almost completely a
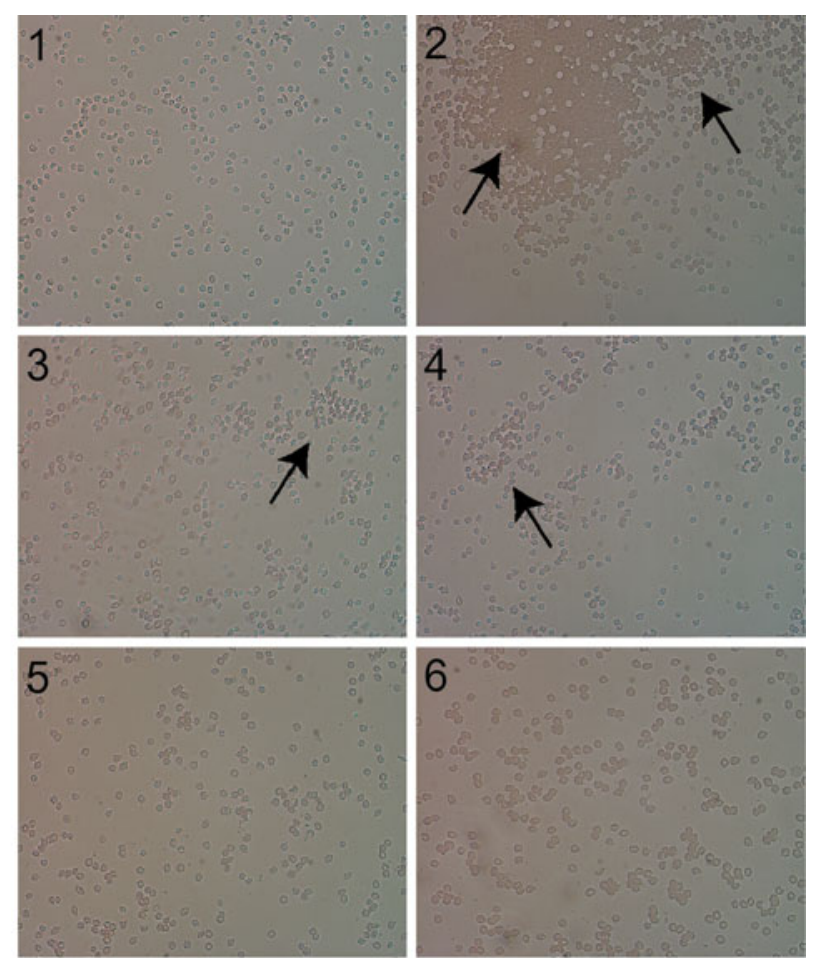

b

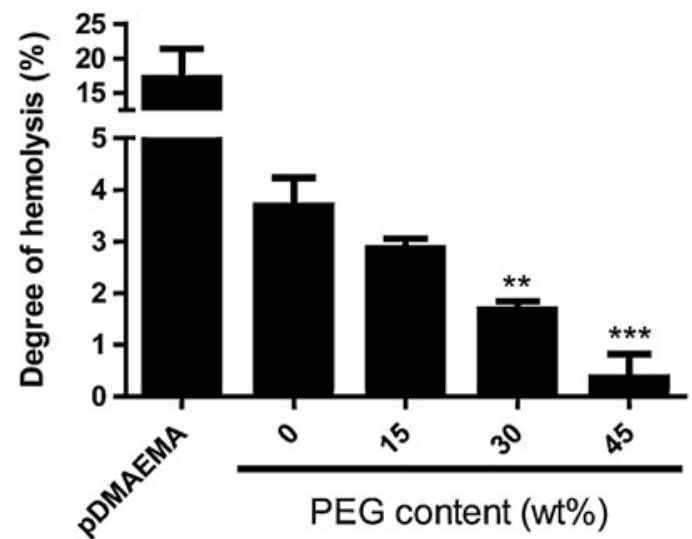

Fig. 7 Polyplex-induced erythrocyte aggregation and hemolysis. Polyplexes were prepared using polymers with increasing PEG content and incubated with erythrocytes for $\mid \mathrm{h}$ at $37^{\circ} \mathrm{C}$. pDMAEMA polyplexes were used as controls. (a) Microscopic image of erythrocyte aggregation. Aggregates are indicated with arrows. (I) HBG (2) pDMAEMA (3-6) p(CBA-ABOL/EDA) with increasing PEG content. (b) Degree of hemolysis as determined by absorbance detection at $550 \mathrm{~nm}$. Results are shown as mean \pm SD for $n=3$. Statistically significant differences versus polyplexes without PEG are denoted by $* *(p<0.01)$ or $* * *(p<0.00 I)$.

inhibited for polyplexes containing 45 wt.\% PEG. Lower cellular uptake of polyplexes having increased PEG content was also confirmed by fluorescent microscopy (Fig. 8b). As suggested by others, cellular uptake for PEGylated polyplexes is reduced because of PEG stealth effects, causing strongly reduced interaction with the cellular membrane $(32,33)$. 
a

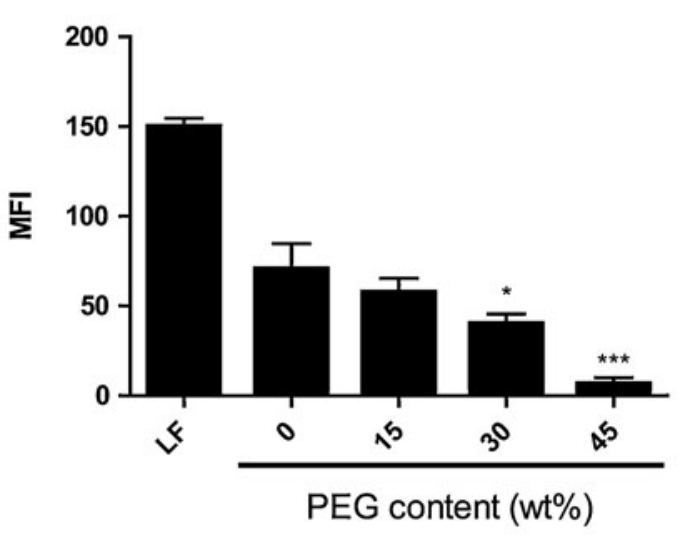

b

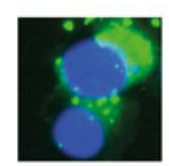

LF

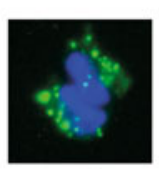

0

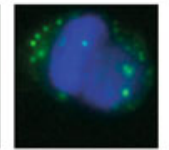

15

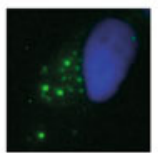

30

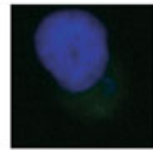

45

\section{PEG content (wt\%)}

Fig. 8 Cellular uptake of PEGylated polyplexes. (a) Mean fluorescence intensity (MFI) of HI 299 cells determined $4 \mathrm{~h}$ after transfection, corrected for protein content. Polyplexes were prepared using polymers with increasing PEG content and fluorescently labeled siRNA. Lipofectamine 2000 was used as control. Results are shown as mean \pm SD for $n=2-3$. Statistically significant differences versus polyplexes without PEG are denoted by $*(p<0.05)$ or $* * * *(p<0.00 \mathrm{I})$. (b) Fluorescent microscopy pictures of HI299 treated cells. Nuclei are stained blue (DAPI), siRNA appears as green (Alexa-488).

Gene silencing potential of the PEGylated polyplexes was evaluated using H1299 cells that stably express the luciferase enzyme. As PEGylation reduced cellular uptake and hence is likely to reduce silencing efficiency, cells were transfected using a rather high siRNA concentration of $125 \mathrm{nM}$. At this concentration, transfection with Lipofectamine 2000 lipoplexes resulted in almost complete luciferase silencing. UnPEGylated polyplexes could efficiently inhibit luciferase expression, however this was combined with a relatively high cytotoxicity (approximately 50\%), as indicated by the reduced luciferase expression of cells treated with control siRNA (Fig. 9). The apparent toxicity of these polyplexes was not observed before $(8,9)$, and is likely caused by the increased polymer and siRNA dose which may lead in the presence of serum to particle aggregation and precipitation. As expected, with increasing PEG content, silencing efficiency was reduced, as well as cytotoxicity. In contrast, others have shown that PEGylation of pEI polyplexes leads to enhanced silencing activity, due to decreased polyplex stability and more efficient siRNA release (23). For our systems, siRNA release from the complex is most likely not a limiting factor for their

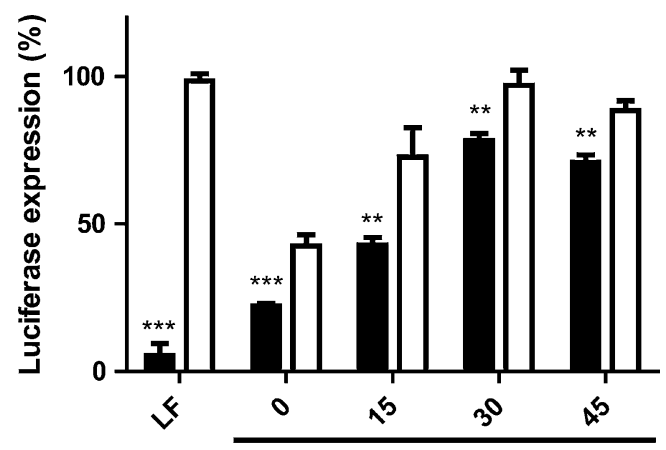

PEG content (wt\%)

Fig. 9 Gene silencing activity of PEGylated polyplexes. Luciferase expression of HI299 cells was determined 48 h after transfection. Polyplexes were prepared using polymers with increasing PEG content and luciferase siRNA (black bars) or non-silencing control siRNA (white bars). Lipofectamine 2000 was used as control. Results are shown as mean \pm SD for $n=3$. Statistically significant differences of luciferase siRNA versus non-silencing control siRNA are denoted by $* * * 0.01)$ or $* * * *(p<0.001)$.

biological activity, as a result of the triggered release mechanism due to the cleavage of the disulfide linkages in the polymer backbone. Interestingly, for polyplexes with high PEG content (30 or 45 wt.\%) cell viability was unaffected, while significant luciferase knockdown was still observed. Although the knockdown efficacy of PEGylated polyplexes in vitro is low as compared to Lipofectamine 2000 or unPEGylated polymers, improved in vivo delivery due to decreased aggregation and increased circulation time can be expected. Addition of targeting ligands to the distal ends of the PEG chains can be expected to boost their cellular uptake, resulting in increased silencing efficiency, and this is considered as the next step in the development of targeted delivery systems for siRNA (34).

\section{CONCLUSIONS}

In this study, the synthesis of a novel PEGylated polymer based on biodegradable poly(amido amine)s with disulfide linkages in the backbone was described for the delivery of siRNA. Polyplexes with various PEG contents were prepared by mixing the PEGylated polymer with its unPEGylated counterpart prior to polyplex formation. PEGylation decreased polyplex surface charge, increased their stability against salt and serum and decreased polyplex interactions with erythrocytes. Controversially, PEGylated polyplexes showed decreased resistance against heparin displacement. Cellular uptake was lower for polyplexes with increasing PEG content, which resulted in reduced gene silencing efficiency, but also reduced toxicity. Polyplexes with PEG contents of 30 and 45 wt.\% showed significant silencing efficiency in the absence of toxicity, which makes 
them promising carriers for delivery of siRNA in vivo. The addition of targeting ligands on the PEG chain ends is expected to further improve their cellular uptake and silencing potential.

\section{ACKNOWLEDGMENTS \& DISCLOSURES}

The authors gratefully acknowledge M.J. van Steenbergen for his assistance with GPC measurements. This project is financially supported by the Technology Foundation STW of The Netherlands Organization for Scientific Research NWO grant UFA7468.

Open Access This article is distributed under the terms of the Creative Commons Attribution Noncommercial License which permits any noncommercial use, distribution, and reproduction in any medium, provided the original author(s) and source are credited.

\section{REFERENCES}

1. de Fougerolles A, Vornlocher HP, Maraganore J, Lieberman J. Interfering with disease: a progress report on siRNA-based therapeutics. Nat Rev Drug Discov. 2007;6:443-53.

2. Schiffelers RM, Woodle MC, Scaria P. Pharmaceutical prospects for RNA interference. Pharm Res. 2004;21:1-7.

3. Howard KA. Delivery of RNA interference therapeutics using polycation-based nanoparticles. Adv Drug Deliv Rev. 2009;61:710-20.

4. Urban-Klein B, Werth S, Abuharbeid S, Czubayko F, Aigner A. RNAi-mediated gene-targeting through systemic application of polyethylenimine (PEI)-complexed siRNA in vivo. Gene Ther. 2005;12:461-6.

5. Sato A, Choi SW, Hirai M, Yamayoshi A, Moriyama R, Yamano $\mathrm{T}$, et al. Polymer brush-stabilized polyplex for a siRNA carrier with long circulatory half-life. J Control Release. 2007;122:20916

6. Howard KA, Rahbek UL, Liu X, Damgaard CK, Glud SZ, Andersen MO, et al. RNA interference in vitro and in vivo using a novel chitosan/siRNA nanoparticle system. Mol Ther. $2006 ; 14: 476-84$.

7. Varkouhi AK, Lammers T, Schiffelers RM, van Steenbergen MJ, Hennink WE, Storm G. Gene silencing activity of siRNA polyplexes based on biodegradable polymers. Eur J Pharm Biopharm. 2011;77:450-7.

8. Vader P, van der Aa LJ, Engbersen JF, Storm G, Schiffelers RM. Disulfide-Based Poly(amido amine)s for siRNA Delivery: Effects of Structure on siRNA Complexation, Cellular Uptake, Gene Silencing and Toxicity. Pharm Res. 2010.

9. van der Aa LJ, Vader P, Storm G, Schiffelers RM, Engbersen JF. Optimization of poly(amido amine)s as vectors for siRNA delivery. J Control Release. 2011;150:177-86.

10. Verbaan FJ, Oussoren C, van Dam IM, Takakura Y, Hashida M, Crommelin DJ, et al. The fate of poly(2-dimethyl amino ethyl) methacrylate-based polyplexes after intravenous administration. Int J Pharm. 2001;214:99-101.

11. Glodde M, Sirsi SR, Lutz GJ. Physiochemical properties of low and high molecular weight poly(ethylene glycol)-grafted poly (ethylene imine) copolymers and their complexes with oligonucleotides. Biomacromolecules. 2006;7:347-56.
12. Ogris M, Brunner S, Schuller S, Kircheis R, Wagner E. PEGylated DNA/transferrin-PEI complexes: reduced interaction with blood components, extended circulation in blood and potential for systemic gene delivery. Gene Ther. 1999;6:595-605.

13. Petersen H, Fechner PM, Martin AL, Kunath K, Stolnik S, Roberts CJ, et al. Polyethylenimine-graft-poly(ethylene glycol) copolymers: influence of copolymer block structure on DNA complexation and biological activities as gene delivery system. Bioconjug Chem. 2002;13:845-54.

14. Choi YH, Liu F, Kim JS, Choi YK, Park JS, Kim SW. Polyethylene glycol-grafted poly-L-lysine as polymeric gene carrier. J Control Release. 1998;54:39-48.

15. Oupicky D, Ogris M, Howard KA, Dash PR, Ulbrich K, Seymour LW. Importance of lateral and steric stabilization of polyelectrolyte gene delivery vectors for extended systemic circulation. Mol Ther. 2002;5:463-72.

16. Hatakeyama $\mathrm{H}$, Akita $\mathrm{H}$, Harashima $\mathrm{H}$. A multifunctional envelope type nano device (MEND) for gene delivery to tumours based on the EPR effect: a strategy for overcoming the PEG dilemma. Adv Drug Deliv Rev. 2011;63:152-60.

17. Brumbach JH, Lin C, Yockman J, Kim WJ, Blevins KS, Engbersen JF, et al. Mixtures of poly(triethylenetetramine/cystamine bisacrylamide) and poly(triethylenetetramine/cystamine bisacrylamide)-g-poly(ethylene glycol) for improved gene delivery. Bioconjug Chem. 2010;21:1753-61.

18. Vader P, van der Aa LJ, Engbersen JF, Storm G, Schiffelers RM. A method for quantifying cellular uptake of fluorescently labeled siRNA. J Control Release. 2010;148:106-9.

19. Lin S, Du F, Wang Y, Ji S, Liang D, Yu L, et al. An acid-labile block copolymer of PDMAEMA and PEG as potential carrier for intelligent gene delivery systems. Biomacromolecules. 2008;9:10915.

20. Germershaus O, Neu M, Behe M, Kissel T. HER2 targeted polyplexes: the effect of polyplex composition and conjugation chemistry on in vitro and in vivo characteristics. Bioconjug Chem. 2008;19:244-53.

21. Merkel OM, Germershaus O, Wada CK, Tarcha PJ, Merdan T, Kissel T. Integrin alphaVbeta3 targeted gene delivery using RGD peptidomimetic conjugates with copolymers of PEGylated poly (ethylene imine). Bioconjug Chem. 2009;20:1270-80.

22. Verbaan FJ, Oussoren C, Snel CJ, Crommelin DJ, Hennink WE, Storm G. Steric stabilization of poly(2-(dimethylamino)ethyl methacrylate)-based polyplexes mediates prolonged circulation and tumor targeting in mice. J Gene Med. 2004;6:64-75.

23. Mao S, Neu M, Germershaus O, Merkel O, Sitterberg J, Bakowsky $\mathrm{U}$, et al. Influence of polyethylene glycol chain length on the physicochemical and biological properties of poly(ethylene imine)-graft-poly(ethylene glycol) block copolymer/SiRNA polyplexes. Bioconjug Chem. 2006;17:1209-18.

24. Bartlettand DW, Davis ME. Physicochemical and biological characterization of targeted, nucleic acid-containing nanoparticles. Bioconjug Chem. 2007;18:456-68.

25. Pun SH, Davis ME. Development of a nonviral gene delivery vehicle for systemic application. Bioconjug Chem. 2002;13:630-9.

26. Buyens K, Lucas B, Raemdonck K, Braeckmans K, Vercammen $\mathrm{J}$. Hendrix J, et al. A fast and sensitive method for measuring the integrity of siRNA-carrier complexes in full human serum. J Control Release. 2008;126:67-76.

27. Ruponen M, Ronkko S, Honkakoski P, PelkonenJ, Tammi M, Urtti A. Extracellular glycosaminoglycans modify cellular trafficking of lipoplexes and polyplexes. J Biol Chem. 2001;276:33875-80.

28. Kircheis R, Wightman L, Schreiber A, Robitza B, Rossler V, Kursa M, et al. Polyethylenimine/DNA complexes shielded by transferrin target gene expression to tumors after systemic application. Gene Ther. 2001;8:28-40. 
29. Verbaan F, van Dam I, Takakura Y, Hashida M, Hennink W, Storm $\mathrm{G}$, et al. Intravenous fate of poly(2-(dimethylamino)ethyl methacrylate)-based polyplexes. Eur J Pharm Sci. 2003;20:419-27.

30. Neu M, Sitterberg J, Bakowsky U, Kissel T. Stabilized nanocarriers for plasmids based upon cross-linked poly(ethylene imine). Biomacromolecules. 2006;7:3428-38.

31. Akagi D, Oba M, Koyama H, Nishiyama N, Fukushima S, Miyata T, et al. Biocompatible micellar nanovectors achieve efficient gene transfer to vascular lesions without cytotoxicity and thrombus formation. Gene Ther. 2007;14:1029-38.
32. Mishra S, Webster P, Davis ME. PEGylation significantly affects cellular uptake and intracellular trafficking of non-viral gene delivery particles. Eur J Cell Biol. 2004;83:97-111.

33. Mok H, Bae KH, Ahn CH, Park TG. PEGylated and MMP-2 specifically dePEGylated quantum dots: comparative evaluation of cellular uptake. Langmuir. 2009;25:1645-50.

34. Schiffelers RM, Ansari A, Xu J, Zhou Q, Tang Q Storm G, et al. Cancer siRNA therapy by tumor selective delivery with ligandtargeted sterically stabilized nanoparticle. Nucleic Acids Res. 2004;32:e149. 